\title{
Preference for Complexity and Asymmetry Contributes to an Ability to Overcome Structured Imagination: Implications for Creative Perception Paradigm
}

\author{
Anatoliy V. Kharkhurin $1, *(1)$ and Morteza Charkhabi ${ }^{2}$ (D) \\ 1 School of Psychology, National Research University Higher School of Economics, 101000 Moscow, Russia \\ 2 Institute of Education, National Research University Higher School of Economics, 101000 Moscow, Russia; \\ mcharkhabi@hse.ru \\ * Correspondence: akharkhurin@hse.ru; Tel.: +7-977-817-0567
}

Citation: Kharkhurin, A.V.; Charkhabi, M. Preference for Complexity and Asymmetry Contributes to an Ability to Overcome Structured Imagination: Implications for Creative Perception Paradigm. Symmetry 2021, 13, 343. https://doi.org/10.3390/ sym 13020343

\section{Academic Editors:}

Chien-Chung Chen, Chiara Spironelli and Tadamasa Sawada

Received: 8 December 2020

Accepted: 12 February 2021

Published: 20 February 2021

Publisher's Note: MDPI stays neutral with regard to jurisdictional claims in published maps and institutional affiliations.

Copyright: (c) 2021 by the authors. Licensee MDPI, Basel, Switzerland. This article is an open access article distributed under the terms and conditions of the Creative Commons Attribution (CC BY) license (https:// creativecommons.org/licenses/by/ $4.0 /)$.

\begin{abstract}
The study is a part of a research project, which explores the role of creative perception in creative behavior. We operationalized creative behavior as an ability to overcome structured imagination, as measured by the Invented Alien Creature test, and operationalized creative perception as a preference for complexity and asymmetry, which we assessed using a standard Barron-Welsh Art Scale. Our group of participants was composed of ninety-three undergraduate students from the United Arab Emirates. The degree to which one preferred complexity and asymmetry measurably contributed to their ability to overcome structured imagination. This finding adds another brick to the rising seventh pillar of the creativity construct, namely, creative perception. The article provides a first sketch of the creative perception paradigm.
\end{abstract}

Keywords: creative perception; creativity; structured imagination; complexity; asymmetry; invented alien creature test; Barron-Welsh Art Scale

\section{Introduction}

The contemporary world is witnessing a dramatic shift towards technocratic hegemony. Our mundane functions have been already successfully replaced by robotic systems. The days are approaching when artificial intelligence will replace human agents as primary decision-makers in most of the economic, social, and cognitive domains. Humanity will be liberated from survival driven urges. What urges will come into their place? Our answer to this question is the human creative act. This makes the need to investigate creative behavior more important than ever.

The current literature on creativity identifies six major constituents of creative behavior, all of which are labeled with P-words. They determine the perspective from which we observe the phenomenon of creativity. Thus, creativity can be studied from the following perspectives: the perspective of a process that triggers creative activity; the perspective of a product that is generated by a creative activity; the perspective of a person engaged in creative activity; the perspective of the set and setting (press) in which a creative activity takes place; the perspective of persuasion and the ability of creative people to influence those around them; and the perspective of creative potential (see [1], for an overview). The present work continues a project aiming at complementing these six Ps with a seventh one, a paradigm investigating creative perception and the way in which it contributes to creative behavior.

From the early days of psychology, human perception was conceptualized as triggering overt behavior (e.g., [2]). Clinical studies demonstrated the significant way in which perception impacted behavior witnessed in epilepsy, aphasia, catatonic states, low-rate mental deficiency, and apraxia (e.g., [3]). Contemporary cognitive and social psychologists claimed that perceptual and behavioral representations are linked and that they share some 
coding systems (e.g., [4]). Recent behavioral and neurophysiological studies supported these claims by providing evidence that action, perception, and their interaction share a common coding (see [5], for a review). If perception contributes to evident behavior, then we anticipate that creative perception likewise contributes to creative behavior.

One of the most elaborated-upon constituents of creative behavior mentioned above (namely, creative process) concerns the cognitive processes that underlay creative thinking. This approach conceives of creative thinking as the capacity to initiate numerous cycles that oscillate between divergent and convergent thinking [6]. These two types of thinking fundamentally differ insofar as convergent thinking is an attention-demanding, conscious process, whereas divergent thinking arises when attention is defocused, and associative thought takes place in the unconscious mind. Convergent thinking approaches a question as though there were one correct solution. In contrast, divergent thinking seeks to generate numerous, novel alternative answers or solutions to a problem which is not considered to have a single solution by engaging in a broader search for information. Convergent thinking is then used to determine a single, optimal solution from the number of possible solutions generated during divergent thinking. Such a combination of divergent and convergent thinking facilitates the generation of ideas satisfying the defining characteristics of a creative product, namely, novelty (i.e., unexpected or original) and utility (i.e., meeting task restrictions or useful) through the creation of an attention-demanding and active process (see [7], for an overview).

The concept of creative perception is vaguely defined and the project in which the present study takes part aims to shed some light in that direction. The project's first study [8] concerned the creative perception of the self. We operationalized perception of the self as creative self-efficacy, meaning the ability of creative people to identify their own creative potential [9]. It revealed that creative self-efficacy is a contributing factor of divergent thinking. Creative self-perception may be considered an inwardly focused mode of perception, directed toward a person's self. The project's second study [10], however, contemplated a contrasting mode of creative perception, one which is directed outwards toward the surrounding environment. It was found to predict divergent thinking as well. Thus, both studies demonstrated that creative perception predicts divergent thinking.

Divergent thinking is widely considered a defining component of the creative process by many researchers [11]. This claim is supported by extensive empirical evidence that divergent thinking tests predict particular aspects of real-world achievements and performance in creative problem-solving. However, it appears to be an oversimplification of the matter to equate divergent with creative thinking. In Runco's words, "Divergent thinking is not synonymous with creative thinking" ([12], p. ix). Hence, to deepen the exploration of the influence of creative perception on creative thinking, the present study focuses on another aspect of creative thinking, namely, an ability to overcome structured imagination.

We reasoned as follows: The ability to generate a multitude of unrelated ideas during divergent thinking seems to rely on a spreading activation mechanism that keeps active multiple unrelated conceptual representations simultaneously [13]. This mechanism appears to facilitate consideration of many properties of different categories at once, which may result in overcoming the standard category boundaries. When people are asked to produce something new, the particular set of characteristics that describe the conceptual category in which this innovation should fit generally restrict their imagination. What they produce is likely to have been based off of the most common set of category properties. Numerous studies in various facets of creative production demonstrated that what people produce is significantly impacted by the semantic structure of a category (e.g., [14]). The 'structured imagination' restricts people's ability to think outside the box; in other words, they reluctantly violate the conceptual borders of the standard category when producing a new and original example of said category. The alien creature invention studies [15] demonstrated that the imperative to violate the boundaries of a standard category enhanced creative thinking (e.g., [14]). Additionally, independent judges rated drawings of the alien creatures with more violations of the standard set of category properties as more 
creative (e.g., [16]). Kozbelt and Durmysheva's [16] coding system classifies the violations of standard conceptual category boundaries in terms of three invariants, that is, features that most participants regularly included in their responses. They demonstrated that the judges' ratings of the drawings based on creativity were significantly and positively correlated with violation of the standard invariants. Therefore, we employed Ward's [15] structured imagination paradigm and operationalized creative thinking in terms of a tendency to overcome the structured imagination.

Similar to Kharkhurin and Yagolkovskiy's study [10], the present study focused on the creative perception of the environment. We consider creative perception of the environment to be a perception that evaluates an individual's ability to discriminate the creative elements of objects or scenes from the non-creative ones. Kharkhurin and Yagolkovskiy [10] argued that this perspective on creative perception is prudent. In a nutshell, perceptual and cognitive approaches to aesthetic activity in the visual arts (e.g., $[17,18])$ concerned themselves with the aesthetic activities that emerge from those perceptual and cognitive processes underlying our everyday experience. To relate creative activity to everyday perception, Gibson [17] differentiated between 'invariances' and 'aspects'. Invariances stem from and rely on constant or stable features, as such features are a necessary and sufficient condition for object identification. In contrast to these constant, stable features, aspects constitute ever-changing, dynamic features; the unique location of a perceiver relative to an object in space-time determines such features. Aesthetic perception appears to be different than everyday perception in at least one way, that is, its tendency to attend to aspects and disregard invariances. It also differentiates those with aesthetic sensitivity from those who lack it. Those without it are predisposed towards searching for identifiable, invariant themes and objects when examining a work of art, whereas those engaged in aesthetic perception appreciate the collage which forms an infinite number of aspects. Artists (those with aesthetic sensitivity) tend to identify aspects in the surrounding world, detect highly informative ones using their knowledge and expectations, and transform their experience of being in the world, of perception, into work of aesthetic value [19]. Consequently, one's aesthetic perception of the environment could instruct their aesthetic action. Regarding the project at hand, the cognitive/perceptual perspective on aesthetic activity raises the possibility that the degree to which one creatively experiences environmental cues might appear a predictor of creative thinking.

It would seem that preference for complexity in perceptual input is one aspect of this creative experience. This assumption is based on Gibson's [17] differentiation of everyday perception from aesthetic perception-the latter of which prefers aspectual complexity - and is in alignment with other theories of aesthetics (see [20], for discussion) that emphasize the critical role that complexity and contradiction play in creative output. As such, it appears that preferring complexity informs aesthetic perception and may be considered a component of creative perception of the environment.

Barron and Welsh [21] developed an assessment tool that measures the degree to which one prefers asymmetry and complexity. Substantial empirical research validated this instrument as a good discriminator between persons high and low in creativity in various literary, artistic, and scientific spheres [22]. It has been found that creative people display a preference for figures of greater complexity and asymmetry as opposed to simpler and more symmetrical ones. We employed the knowledge of this preference in the present study as an operational definition of the creative perception of the environment. Indication of such a preference for complex and asymmetrical perceptual elements is interpreted as a more creative perception of the environment.

The plausibility of relating creative perception of the environment to the ability to overcome structured imagination was suggested by a parallel between invariants in Ward's [15] structured imagination paradigm and the distinction Gibson [17] made between invariances and aspects, as discussed above. Invariants represent standard features of a category. The habit of attending to invariances represents non-creative perception. The tendency to violate the invariants is regarded as an ability to think outside the box; that is, creative 
thinking. The tendency to disregard invariances and attend to aspects is considered as a characteristic of creative perception of the environment. Hence, we hypothesized that creative perception of the environment would predict violation of invariants. In line with this hypothesis, we expected to find that preference for complexity and asymmetry would predict performance on structured imagination test.

Further, as per the discussion above, creative thinking involves both divergent and convergent thinking. The latter appears to be closely related to intelligence [23]. The evaluation process inherent to convergent thinking largely relies on an individual's knowledge base because the alternatives generated during divergent thinking should be compared with the previously generated solutions to ensure the originality. An individual's knowledge provides a database against which the new ideas are assessed. An ability to manipulate these ideas to generate novel solutions to a problem may stem from intelligence as well. The two types of intelligence, one related to an individual's knowledge and the other to an ability to manipulate this knowledge, have been defined by Cattell [24] as crystallized and fluid intelligence, respectively. The latter involves solving novel, complex problems through reasoning inductively and deductively, and engaging in classification and concept formation; it involves the employment of effective reasoning in novel situations. The former refers to knowledge already acquired, partially through fluid intelligence, from culture, education, and other experiences.

The relationship between intelligence and convergent thinking was extensively discussed in the literature (see [25], for an overview). Runco and colleagues [23] even argue that the tests of intelligence in fact measure an individual's convergent thinking. This is a quite controversial perspective, however, as research provides mixed findings on the relationship between creativity and intelligence, ranging from the view that there exists no relation between the two to the view that one is a subset of the other. Nevertheless, a potential overlap in cognitive functioning between convergent thinking and intelligence should not be withdrawn from an account.

Thus, on one side, convergent thinking appears to be constituted by creative thinking, and on the other, it appears to be related to intelligence. Hence, we believe that the exploration of the influence of creative perception on creative thinking should account for the effect of intelligence. We employed Cattell's [24] fluid intelligence as a potential contributor to creative thinking. A large number of empirical studies assessing fluid intelligence utilize a standard Culture Fair Intelligence Test battery [26] that uses nonverbal stimuli in order to try and mitigate the influence of verbal fluency, culture, and educational level as much as possible when assessing intelligence.

Altogether, our study explores the relationship between creative perception and creative thinking by looking at how the preference for complexity and asymmetry and fluid intelligence predict the ability to overcome structured imagination.

\section{Materials and Methods}

\subsection{Participants}

We recruited ninety-three American University of Sharjah (United Arab Emirates) undergraduate students ( 34 male and 59 female) aged between 16 and $23(M=20.02, S D=1.59)$ using a simple random sampling method to participate in this study. The sample size was calculated using the $G^{*}$ Power statistical package (https:/ / www.psychologie.hhu.de/ arbeitsgruppen/allgemeine-psychologie-und-arbeitspsychologie/gpower.html, accessed on 27 September 2015) [27]. Considering that the total number of undergraduate students enrolled in the College of Arts and Sciences in the testing period (Spring 2013) was 670 (for a statistical review: https: / / www.aus.edu/about/aus-at-a-glance/facts-and-figures / fastfacts-spring-2013, accessed on 13 November 2020) our sample was convenient. Moreover, this sample size was comparable with other studies that used a similar procedure (e.g., 65 in [8], and 139 in [10]). 


\subsection{Procedure}

We invited participants to join in the study through the Introduction to Psychology subject pool powered by the SONA platform (https: / / www.sona-systems.com/default. aspx, accessed on 4 June 2018). More specifically, they received an invitation letter from our research team containing information about the study, research aims, compensation, and instruction on how to take part in this study. They had the choice to accept or reject participation in the study. They were informed about the voluntary nature of this study and their right to disengage from the study at any point. Participants did not take any art or creativity course before testing. Thus, students were not introduced to any concept of symmetry/asymmetry and the related impact on creativity. If accepted to participate, they were asked to sign an official consent form authorized by the American University of Sharjah Institutional Review Board. The participants were given a course credit as compensation for their participation in the study.

The testing took place in the laboratory space in a group of 20 participants. Participants received a structured imagination test followed by a test of preference for complexity and asymmetry and a test of fluid intelligence (in an attempt to prevent a fatigue effect, the order of the last two were counterbalanced). Each test was preceded with an oral instruction provided by the experimenter. All tests were standard assessment tools and used the procedure and timing provided in the test manual. The first and third tests were timed (12 and $12.5 \mathrm{~min}$, respectively). The second test was not timed; on average, participants spent 5-7 min doing it.

\subsection{Measures}

\subsubsection{The Test of Structured Imagination}

We measured structured imagination using the Invented Alien Creatures Task, albeit a modified version ([16]; cf. [15]). The procedure was abridged from the original in order to match the purpose of the present study. We instructed participants to use their imagination to describe and draw a creature from beyond Earth. They were instructed to think as creatively and imaginatively as possible, and to be less concerned about the artistic qualities of their drawing. They were given $12 \mathrm{~min}$ to finish the task.

An invariant coding system [16] was utilized to categorize each drawing on three invariants (the traits that normally appeared among the participants' drawings): two eyes, four limbs, and bilateral symmetry. The selected invariants resembled those identified during Ward's [15] original study that revealed the fact that "the majority of imagined creatures were structured by properties that are typical of animals on earth: bilateral symmetry, sensory receptors, and appendages" (p. 1).

Each invariant was rated using assigned values, indicated here in parentheses. The categories for bilateral symmetry included undoubted bilateral symmetry (0), bilateral symmetry if the creature were rotated (0), superficial violation of bilateral symmetry (e.g., an additional limb or appendage on one side; 1$)$, undoubted absence of bilateral symmetry (2), and those responses which were unclear in regard to this invariant (0). The standard presence of two eyes and four limbs were assigned values based on the following criteria: does not deviate from the invariant (possesses two eyes and/or four limbs; 0), depicting a greater number of features than the invariant (exceeding two eyes or four limbs; 2), drawing fewer features than the invariant (for example, drawing only one eye or three limbs; 2), drawing no such features whatsoever (1), and once again, those responses which are unclear in this regard (0). Altogether, every drawing obtained an invariant score ranging from 0 (not violated) to 2 (clearly violated). Subsequently, the total invariants violation (IV) score was computed as a total of three invariants scores. The IV score ranged from 0 to 6; a higher score suggested a greater tendency to violate the standard invariants in the drawing. Figure 1 presents two cases illustrating (a) no violation of invariants and (b) some violation of invariants. An 'alien puppy' (Figure 1a) is bilaterally symmetric (score 0), has two eyes (score 0 ) and four limbs (score 0 ); its IV $=0$. The creature in Figure $1 \mathrm{~b}$ is bilaterally symmetric (score 0 ), has more than two eyes (score 2) and two limbs (score 2); its IV = 4 . 


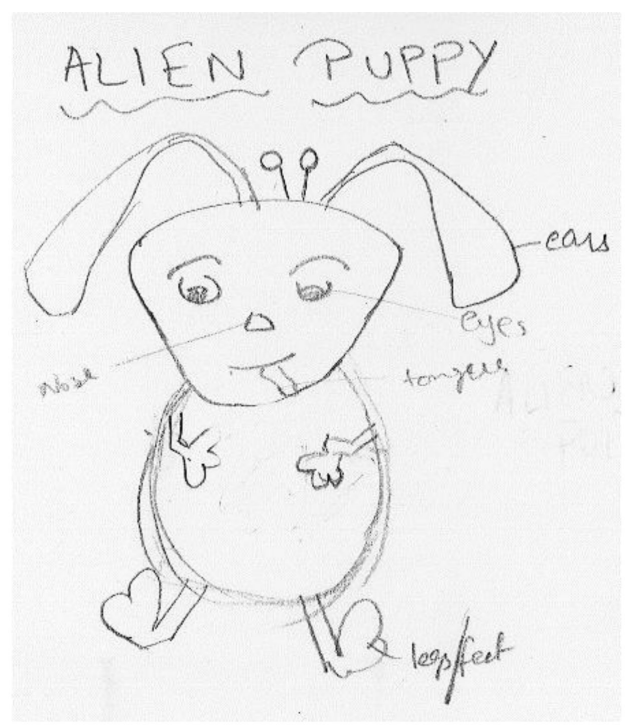

(a)

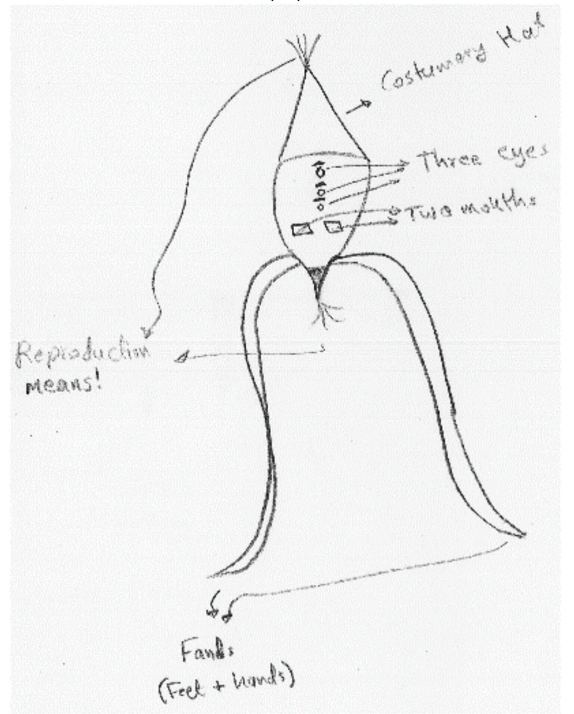

(b)

Figure 1. Examples of the alien creatures produced in the Invented Alien Creatures Task, which received $(\mathbf{a})$ low $(\mathrm{IV}=0)$ and $(\mathbf{b})$ high $(\mathrm{IV}=4)$ invariant violation scores, respectively.

Two independent assessors rated the invariants violation of participants using the Invented Alien Creatures task's rating procedure described above. The evaluation of the invariant violation scores obtained from those raters resulted in a consistency of $\rho=0.78$ $(p<0.001)$. Such comparability of the ratings indicates that the assessors followed similar rationales. The scores produced by the two raters were then averaged to calculate the IV score.

\subsubsection{The Test of Preference for Complexity and Asymmetry}

The degree to which participants appeared to express a preference for complexity and asymmetry (PCA) was assessed using a Barron-Welsh Art Scale (BWAS; [28]). It consists of 86 black and white drawings with varying complexity and symmetry. These 86 items were selected from among the 400 figures that comprise the Welsh Figure Preference Test [29], and included 24 complex and/or asymmetrical figures which artists favored, 38 simple and/or symmetrical figures which were not favored by artists (in combination, these 62 figures were best able to distinguish control subjects from artists), and 24 filler figures that were not considered during scoring. Participants were prompted to indicate 
whether they liked or disliked the drawings. According to the procedure for scoring this test, one point was given for liking complex and asymmetrical patterns or disliking simple and symmetrical patterns; the final score is calculated as the sum of the 62 figures, meaning it can range from 0 to 62 . Considering this, the PCA score is an attempt to quantify one's preference for more complex and asymmetrical elements over simpler and more symmetrical elements.

The BWAS was found to correlate significantly with other methods used to measure creative talent (e.g., [28]). The responses which we received are consistent within nonclinical samples, maintaining a reliability greater than or equal to 0.90 [22].

\subsubsection{The Test of Fluid Intelligence}

We assessed the fluid intelligence (Gf) of participants using the standard Culture Fair Intelligence Test (CFIT; [26]). The present study utilized Scale 3 Form A of the CFIT in particular. The scale consists of four subtests containing various perceptual tasks. Thus, as a whole, this measure of intelligence is not reliant upon a single skill. An experimenter articulated the instructions for each subtest. These instructions were followed by examples to ensure that participants understood the task requirements. The first of the four subtests was the Series subtest, in which participants were presented with an incomplete, progressive series and tasked with selecting a figure which best continued the series from a collection of possible answers. Second, in the Classification subtest, participants were presented with five figures and asked to correctly identify which two deviated from the other three. During the third, the Matrices subtest, the participants were presented with a matrix and tasked with correctly completing its design. Finally, in the Conditions subtest, participants were given five choices and tasked with selecting which one best duplicated the conditions given in the leftward box.

After summarizing the raw scores which we obtained from each of the four subtests, we transformed them, in accordance with the recommended procedure, into a normalized Gf score [26]. This procedure accounted for age-related norms. According to the CFIT manual, the reliability coefficients for consistency over items is 0.74 , test-retest consistency is 0.69 , concept validity is 0.85 , and concrete validity (calculated as a direct correlation with other tests of intelligence) is 0.66 .

\section{Results}

Figure 2 shows the conceptual model of the relations between research variables in the present study. We looked at the prediction of invariant violation by preference for complexity and asymmetry and fluid intelligence with and without the moderating effect of gender and age.

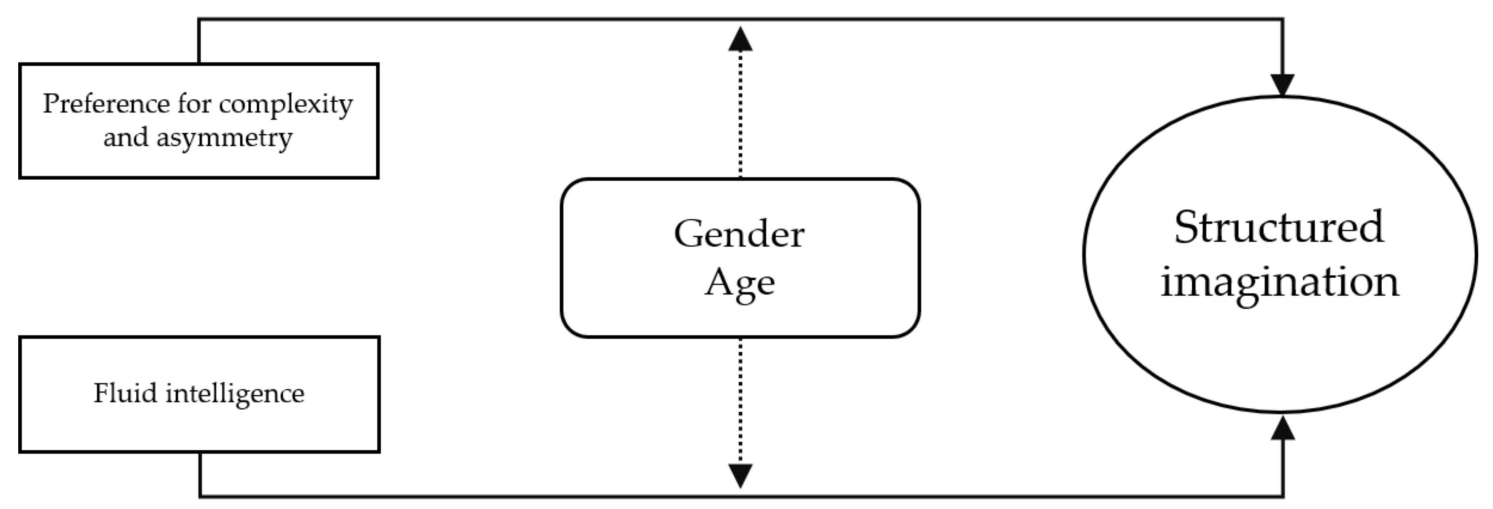

Figure 2. A conceptual model of the association between research variables.

Descriptive statistics (mean and standard deviation) as well as the correlation matrix between the predictors and outcome variables in the present study can be viewed below in Table 1. As the table shows, there is a significant positive correlation between our 
independent variable (fluid intelligence) and our dependent variable (invariant violation) $(r=0.209, p<0.05)$. Similarly, preference for complexity and asymmetry, our second independent variable, is also significantly associated with invariant violation $(r=0.334$, $p<0.01$ ). Although both fluid intelligence and preference for complexity and asymmetry have a significant association with invariant violation, the preference for complexity and asymmetry-invariant violation relationship was found to be stronger.

Table 1. Correlation matrix between research variables in the present study $(n=93)$.

\begin{tabular}{|c|c|c|c|c|c|c|c|c|}
\hline & $M$ & $S D$ & 1 & 2 & 3 & 4 & 5 & 6 \\
\hline 1. Gf & 106.950 & 14.870 & - & & & & & \\
\hline 2. PCA & 32.247 & 12.076 & 0.084 & - & & & & \\
\hline 3. Bilateral symmetry & 0.247 & 0.387 & -0.055 & 0.102 & - & & & \\
\hline 4. Eyes & 0.629 & 0.875 & 0.028 & $0.06 \overline{7}$ & -0.039 & - & & \\
\hline 5. Limbs & 0.742 & 0.855 & -0.008 & $0.228 *$ & 0.145 & 0.194 & - & \\
\hline 6. IV & 1.59 & 1.48 & $0.209 *$ & $0.334^{* *}$ & 0.183 & $0.469 * *$ & 0.208 * & - \\
\hline
\end{tabular}

Further, we looked at the effect of gender on structured imagination. The reason for the inclusion of this variable stems from a considerable controversy with respect to the gender differences in creativity. For example, a capsulization of the current psychological and neuroscientific literature [30] provided mixed findings regarding gender differences in creative cognition; they proposed that one's cognitive style in creative problem-solving could be gender dependent.

In the following paragraphs, we demonstrate the graphical distribution of predictors and outcome variables across gender. Figure 3 illustrates the distribution of invariant violation and its three components across gender. As the figure shows, men reported slightly higher total creative behavior than women did. However, the $t$-test did not detect any significant difference between the two groups. In addition, although women performed better in limbs component than men, the results of the $t$-test revealed that the two groups do not differ from one another in a significant way.

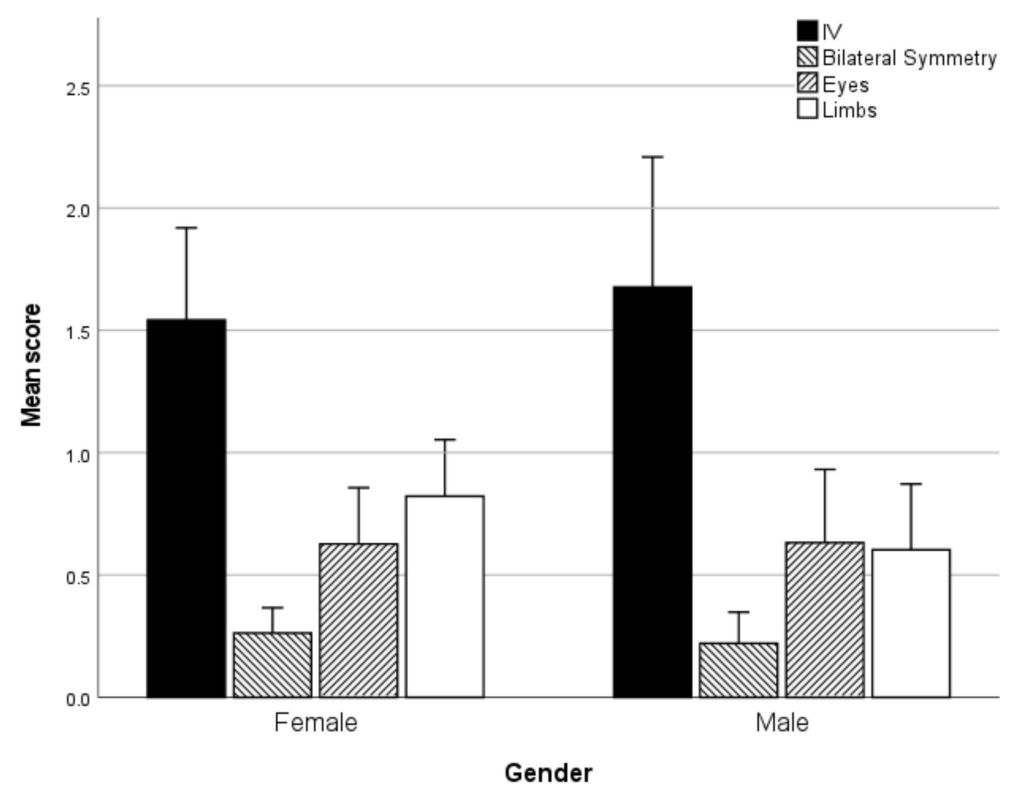

Figure 3. Distribution of invariant violation (IV) and its component across gender (female: $n=59$; male: $n=34)$.

Figure 4 illustrates both preference for complexity and asymmetry and fluid intelligence as the predictors of invariant violation. As the figure shows, although there might be a slight difference between men and women in preference for complexity and asymmetry and fluid intelligence, the difference was not confirmed after we ran the test. 


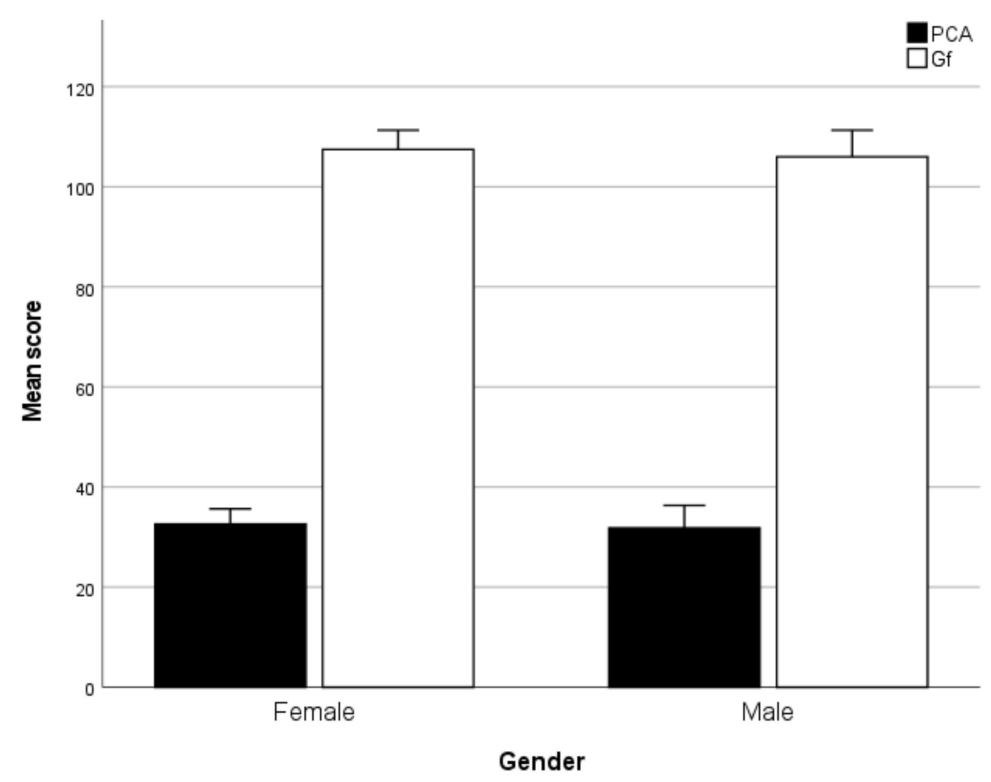

Figure 4. Distribution of preference for complexity and asymmetry (PCA) and fluid intelligence (Gf) as the predictors of invariant violation across gender (female: $n=59$; male: $n=34$ ).

To explore the causal relations between the research variables further, we ran a regression analysis. We have included the results of said analysis in Table 2 (see below). Referring to the table, of the two predictors, only preference for complexity and asymmetry predicated the invariant violation $(\beta=0.039, p<0.01)$. Moreover, the results of separate regression test in male and female groups showed that although preference for complexity and asymmetry significantly predicts the invariant violation in both groups, it is found to be a stronger predictor of invariant violation in males $(\beta=0.099, p<0.01)$ rather than in females $(\beta=0.032, p<0.05)$. We also tested the extent to which demographic variables such as age and gender can predict the invariant violation. As Table 2 displays, none of these variables predicts invariant violation. Furthermore, we tested the possible interaction effects of age and gender in the link preference for complexity and asymmetry-invariant violation and in the link fluid intelligence-invariant violation, but none of the interactions was significant.

Table 2. Results of regression analysis between research variables in the present study $(n=93)$.

\begin{tabular}{|c|c|c|c|c|c|c|}
\hline & & $\begin{array}{r}\text { Unstanda } \\
B\end{array}$ & $\begin{array}{l}\text { oefficients } \\
\text { pr }\end{array}$ & Standardized Coefficients Beta & $t$ & Sig. Level \\
\hline \multirow{3}{*}{ Total $(n=93)$} & Constant & -1.612 & 1.101 & & -1.464 & 0.147 \\
\hline & PCA & 0.039 & $\begin{array}{l}1.101 \\
0.012\end{array}$ & 0.317 & 3.221 & $0.002 * *$ \\
\hline & Gf & 0.018 & 0.010 & 0.183 & 1.855 & 0.067 \\
\hline \multirow{3}{*}{ Males $(n=34)$} & Constant & -2.344 & 1.771 & & -1.323 & 0.196 \\
\hline & PCA & 0.049 & 0.019 & 0.405 & 2.517 & 0.017 * \\
\hline & Gf & 0.023 & 0.017 & 0.227 & 1.414 & 0.168 \\
\hline \multirow{3}{*}{ Females $(n=59)$} & Constant & -1.104 & 1.440 & & -0.767 & 0.447 \\
\hline & PCA & 0.032 & 0.016 & 0.258 & 2.023 & $0.048 *$ \\
\hline & Gf & 0.015 & 0.013 & 0.152 & 1.195 & 0.237 \\
\hline \multirow{3}{*}{ Demographic $(n=93)$} & Constant & 1.941 & 1.978 & & 0.981 & 0.329 \\
\hline & Age & -0.020 & 0.099 & -0.022 & -0.202 & 0.840 \\
\hline & Gender & 0.144 & 0.325 & 0.047 & 0.442 & 0.660 \\
\hline
\end{tabular}

Notes: Dependent Variable: structured imagination; ${ }^{*}$ significant at the 0.05 level; ${ }^{* *}$ significant at the 0.01 level.

\section{Discussion}

Through this study, we have continued to explore the possibility that creative perception contributes to creative behavior in the context of creative thinking. Creative perception of the environment (operationalized as preference for complexity and asymmetry) was found to predict the creative thinking (operationalized as the ability to overcome struc- 
tured imagination). The tendency to search for invariances in one's surroundings seems to encourage the structured imagination. That is, those people would reluctantly violate the boundaries of a standard, conceptual category when prompted to create a new example within said category (see discussion of the structured imagination in the beginning of the article). They would preserve the invariants in producing an alien creature in the test of structured imagination. At the same time, individuals with greater creative perception of the environment would deliberately avoid the invariants and look for unconventional forms of expression. They would more readily violate the standard properties of conceptual categories, and consequently produce alien creatures with fewer invariants.

Further, the finding of the positive significant relationship between the performance on the Alien Creature Test and the BWAS provides evidentiary support for the latter's construct. The BWAS manual [28] reported the findings that "scores on the [test] were shown to relate significantly to ratings of creativity, whether based on observable products and performance or on personal style and expressive behavior" (p. 22). This test's efficacy is further described in its manual, which provides a number of previous studies in which this test correlated with ratings of creativity in 22 samples of students, creative, and non-creative professionals. However, these correlations were relatively low (the median coefficient was 0.23 and only 10 of these coefficients reached significance). Furnham and Bachtiar [31] also found a significant, positive correlation between this test and a variation of the unusual uses test (in which participants create a list of as many unusual uses of a mundane object like a paperclip, blanket, pencil as possible in a given timeframe) administered to private school students; again, the correlation was relatively low $(r=0.22)$. Only Kharkhurin and Yagolkovskiy [10] reported a relatively high correlation $(r=0.35)$ between this test and the test of divergent thinking in a study with college students.

Additionally, we uncovered a positive, significant relationship between performance on the Culture Fair Intelligence Test and performance on the Alien Creature Task. This finding contributes to the aforementioned debate regarding the connection between intelligence and creativity. Those who have looked into this relationship have found low, mediocre and relatively high correlations alike (see [25], for a review). Some researchers even claim that this relationship can take any direction (e.g., [32]). Most studies in this area of research looked into the relationship between fluid intelligence and creative potential (measured by divergent thinking tests; [33]; for review see [34]) and on average reported a correlation of around $r=0.20$. We found the same correlation between fluid intelligence and an ability to overcome structured imagination.

Finally, neither age nor gender was found to have a measurable effect on the relationship between creative perception and creative behavior. The former finding is obvious because we tested a relatively homogenous group of students. Although, their age varies from 16 to 23, this variation appears to not influence the relationship between creative perception and creative thinking. The latter finding suggests that the contribution of the creative perception to the creative behavior does not vary across gender. This is another contribution to a large body of findings demonstrating no significant differences in creative capacities between males and females (although, other mixed findings hint at superior creative abilities in females; review in [30]).

The present work along with two previous studies $[8,10]$ continued examining the concept of creative perception. At this point, it seems to be appropriate to elaborate on the definition of this concept. So far, we found that creative self-efficacy and preference for complexity and asymmetry contribute to the expression of creative potential (measured by divergent thinking test; see [33]) and overcoming structured imagination in thinking. These two cognitive processes were conceptualized as creative perception of the self and the environment, respectively. What do they have in common? Both processes refer to an ability to organize and interpret information in a way that triggers creative thinking. In the first case, one analyzes own personality traits, and in the second one analyzes the sensory information coming from the environmental input. In both cases, this ability depends on the identification of creative elements, either in the self or in the surrounding. In the same 
vein, one can attend to the creative personality traits and/or behavior of the other person; that is, being able to identify creative elements in the others. Hence, creative perception can be defined as an individual's ability to identify creative elements in oneself, others, and the environment. This ability may encourage an individual to engage in creative thinking and subsequently in creative behavior.

In fact, many creative people would make an even stronger claim that creative thinking starts with creative perception. This idea finds support in the anecdotal evidence reported by artists, poets, and people of other creative professions as well as by the first author's own artistic and literary practices. First, we notice creative elements, and then we contemplate on our observations. This process of contemplation initiates creative thinking.

What are these creative elements? Kharkhurin's [20] conceptualization of the aesthetic value of creative work offers a possible answer to this question. He asserts that "The aesthetics attribute stipulates that a creative work presents the fundamental truth of nature, which is reflected in a perfect order, efficiently presents the essence of the phenomenal reality, and is satisfactorily complex, expressing both tension and intrinsic contradiction" (p. 338). Hence, the creative elements in question are those essential constituents of phenomenal reality that reflect the fundamental truth of nature. The ability to identify these constituents encourages an individual to engage in the process of expressing them in one's creative act; that is, in creative thinking and subsequently creative behavior.

We identified the first candidate for those creative elements: complexity, which corresponds to Kharkhurin's understanding of a "satisfactorily complex" creative work. The prudence of selecting complexity as a perceptual creative element is supported by research in aesthetics. One such example comes from Vygotsky [35], who argued that one's aesthetic reaction to a work of art increases because of its complication. He believed that the presence of multiple interpretive standpoints serves to complicate plots, provoke conjectures, stimulate curiosity, and divide attention. The significant role of complexity in creative work was emphasized by Arnheim [36]. Meaning is frequently complex, and any creative endeavor should take this into account. He believed that tension and complexity are structurally integral to the wholeness of a work of art. Berlyne placed complexity at the core of his new experimental aesthetics [37]. According to his theory of aesthetic response, a beholder's pleasure in response to an object will increase with increased complexity. Contemporary research in empirical aesthetics also provides evidence demonstrating that stimulus complexity appears to be an important determinant of aesthetic preference (e.g., [38,39]).

At the same time, complexity appears to be inherent to creative behavior. Runco [1] called creativity a complex. Divergent thinking [6] implies simultaneous consideration of multiple conceptual plains. Aforementioned studies [10,21,31] demonstrated a relationship between preference for complexity and various creativity measures. Preference for complexity appears to be an important creative personality trait. Last, but not least, Barron's transdisciplinary work (e.g., [40]) pointed to the strong connection between complexity and creativity. He argued that research into creativity can help advise the application of complexity theory in the human context.

\section{Limitations}

The present study has some limitations we would like to address. First, at least some of the assessment tools we used to collect data were self-administrated. Although our research team provided participants with clear instructions and answered their questions, some participants may still have responded based on social desirability, which may have threatened the internal validity of this study. We suggest future studies to use multimethods to track the performance of participants over time. Second, we use a convenience sample size instead of a representative sample size that may limit the generalizability of our findings to other samples. This was mainly because the tasks were considered timeconsuming for students to complete and we did not have financial rewards to compensate their efforts. Future research may include a larger sample to establish the reliability of the effects found in this study. 


\section{Conclusions}

Our findings signify the onset of a long and intricate search for elements of creative perception. It is evidently premature, however, to draw any far-reaching conclusions at the present moment. That being said, the creative perception paradigm has the potential to broaden the horizons of the study of creativity as a measurable, observable phenomenon, and to complement the 6Ps approach mentioned in the introduction with the seventh $\mathrm{P}$ for perception.

Author Contributions: All authors have read and agreed to the published version of the manuscript.

Funding: The article was prepared in the framework of a research grant funded by the Ministry of Science and Higher Education of the Russian Federation (grant ID: 075-15-2020-928).

Institutional Review Board Statement: The study was conducted according to the guidelines of the Declaration of Helsinki, and approved by the Institutional Review Board of American University of Sharjah (protocol code 183 and date of approval 24 May 2012).

Informed Consent Statement: Informed consent was obtained from all subjects involved in the study.

Data Availability Statement: The data presented in this study are available on request from the corresponding author. The data are not publicly available due to privacy reasons.

Acknowledgments: The authors thank Ethan Holmes for editing the final draft of the article.

Conflicts of Interest: The authors declare no conflict of interest.

\section{References}

1. Runco, M.A. Creativity: Theories and Themes: Research, Development, and Practice, 2nd ed.; Elsevier Academic Press: Boston, MA, USA, 2014.

2. James, W. The Principles of Psychology; Harvard University Press: Harvard, MA, USA, 1890.

3. Prinz, W. Perception and Action Planning. Eur. J. Cogn. Psychol. 1997, 9, 129-154. [CrossRef]

4. Berkowitz, L. Some effects of thoughts on anti- and prosocial influences of media events: A cognitive-neoassociation analysis. Psychol. Bull. 1984, 95, 410-427. [CrossRef] [PubMed]

5. Creem-Regehr, S.H.; Kunz, B.R. Perception and action. Wiley Interdiscip. Rev. Cogn. Sci. 2010, 1, 800-810. [CrossRef]

6. Guilford, J.P. The Nature of Human Intelligence; McGraw-Hill: New York, NY, USA, 1967.

7. Mayer, R.E. Fifty years of creativity research. In Handbook of Creativity; Sternberg, R.J., Ed.; Cambridge University Press: New York, NY, USA, 1999; pp. 449-460.

8. Kharkhurin, A.V. Does the Eye of the Beholder Construct Beauty? Contributions of Self-Efficacy Factors to Divergent Thinking Traits. Creat. Res. J. 2017, 29, 370-376. [CrossRef]

9. Tierney, P.; Farmer, S.M. Creative Self-Efficacy: Its potential antecedents and relationship to creative performance. Acad. Manag. J. 2002, 45, 1137-1148. [CrossRef]

10. Kharkhurin, A.V.; Yagolkovskiy, S.R. Preference for Complexity and Asymmetry Contributes to Elaboration in Divergent Thinking. Creat. Res. J. 2019, 31, 342-348. [CrossRef]

11. Lubart, T.I. Models of the Creative Process: Past, Present and Future. Creat. Res. J. 2001, 13, 295-308. [CrossRef]

12. Runco, M.A. Divergent Thinking; Ablex Publishing: Westport, CT, USA, 1991.

13. Kharkhurin, A.V. The role of bilingualism in creative performance on divergent thinking and Invented Alien Creatures tests. J. Creat. Behav. 2009, 43, 59-71. [CrossRef]

14. Ward, T.B.; Patterson, M.J.; Sifonis, C.M.; Dodds, R.A.; Saunders, K.N. The role of graded category structure in imaginative thought. Mem. Cogn. 2002, 30, 199-216. [CrossRef]

15. Ward, T. Structured Imagination: The Role of Category Structure in Exemplar Generation. Cogn. Psychol. 1994, 27, 1-40. [CrossRef]

16. Kozbelt, A.; Durmysheva, Y. Understanding creativity judgments of invented alien creatures: The roles of invariants and other predictors. J. Creat. Behav. 2007, 41, 223-248. [CrossRef]

17. Gibson, J.J. The information available in pictures. Leonardo 1971, 4, 27-35. [CrossRef]

18. Hochberg, J. Some of the things that paintings are. In Perception and Pictorial Representation; Nodine, C.F., Fisher, D.F., Eds.; Praeger: New York, NY, USA, 1979; pp. 17-41.

19. Cupchik, G.C. From perception to production: A multilevel analysis of the aesthetic process. In Emerging Visions of the Aesthetic Process: Psychology, Semiology, and Philosophy; Cupchik, G.C., László, J., Eds.; Cambridge University Press: New York, NY, USA, 1992; pp. 83-99.

20. Kharkhurin, A.V. Creativity.4in1: Four-Criterion Construct of Creativity. Creat. Res. J. 2014, 26, 338-352. [CrossRef]

21. Barron, F.; Welsh, G.S. Artistic Perception as a Possible Factor in Personality Style: Its Measurement by a Figure Preference Test. J. Psychol. 1952, 33, 199-203. [CrossRef] 
22. Gough, H.G.; Hall, W.; Bradley, P. Forty years of experience with the Barron-Welsh Art Scale. In Unusual Associates: A Festschrift for Frank Barron; Hampton Press: Cresskill, NJ, USA, 1996; pp. 252-301.

23. Runco, M.A.; Dow, G.; Smith, W.R. Information, Experience, and Divergent Thinking: An Empirical Test. Creat. Res. J. 2006, 18, 269-277. [CrossRef]

24. Cattell, R.B. Theory of fluid and crystallized intelligence: A critical experiment. J. Educ. Psychol. 1963, 54, 1-22. [CrossRef]

25. Kim, K.H.; Cramond, B.; VanTassel-Baska, J. The relationship between creativity and intelligence. In The Cambridge Handbook of Creativity; Kaufman, J.C., Sternberg, R.J., Eds.; Cambridge University Press: New York, NY, USA, 2010; pp. $395-412$.

26. Cattell, R.B. Manual for the Cattell Culture Fair Intelligence Test; Institute for Personality and Ability Testing: Champaign, IL, USA, 1973.

27. Faul, F.; Erdfelder, E.; Buchner, A.; Lang, A.-G. Statistical power analyses using G*Power 3.1: Tests for correlation and regression analyses. Behav. Res. Methods 2009, 41, 1149-1160. [CrossRef]

28. Barron, F.; Welsh, G.S. Barron-Welsh Art Scale; Mind Garden: Menlo Park, CA, USA, 2005.

29. Welsh, G.S. Manual for the Welsh Figure Preference Test; Consulting Psychologists Press: Palo Alto, CA, USA, 1980.

30. Abraham, A. Gender and creativity: An overview of psychological and neuroscientific literature. Brain Imaging Behav. 2016, 10, 609-618. [CrossRef]

31. Furnham, A.; Bachtiar, V. Personality and intelligence as predictors of creativity. Pers. Individ. Differ. 2008, 45, 613-617. [CrossRef]

32. Deary, I.J. Intelligence. Annu. Rev. Psychol. 2012, 63, 453-482. [CrossRef]

33. Runco, M.A. Divergent thinking, creativity, and ideation. In The Cambridge Handbook of Creativity; Cambridge University Press: New York, NY, USA, 2010; pp. 413-446.

34. Kaufman, J.C.; Plucker, J.A. Intelligence and creativity. In The Cambridge Handbook of Intelligence; Cambridge University Press: New York, NY, USA, 2011; pp. 771-783.

35. Vygotsky, L.S. Psychology of Art; MIT Press: Cambridge, MA, USA, 1925. (In Russian)

36. Arnheim, R. The Dynamics of Architectural Form; University of California Press: Berkeley, CA, USA, 1977.

37. Berlyne, D.E. (Ed.) Studies in the New Experimental Aesthetics: Steps Toward an Objective Psychology of Aesthetic Appreciation; Hemisphere Publishing Corporation: Washington, DC, USA, 1974.

38. Lavdas, A.A.; Schirpke, U. Aesthetic preference is related to organized complexity. PLoS ONE 2020, 15, e0235257. [CrossRef] [PubMed]

39. Delplanque, J.; De Loof, E.; Janssens, C.; Verguts, T. The sound of beauty: How complexity determines aesthetic preference. Acta Psychol. 2019, 192, 146-152. [CrossRef] [PubMed]

40. Barron, F. No Rootless Flower: Towards an Ecology of Creativity; Hampton Press: Cresskill, NJ, USA, 1995. 